\title{
IMPACT OF BUCCAL GLUCOSE SPRAY, LIQUID SUGARS AND DEXTROSE TABLETS ON THE EVOLUTION OF PLASMA GLUCOSE CONCENTRATION IN HEALTHY PERSONS
}

\author{
Rudolf Chlup a, b, Jana Zapletalovac ${ }^{c}$ Karolina Peterson ${ }^{a}$, Iveta Poljakova ${ }^{a}$, Eva Lenhartova ${ }^{a}$, \\ Adam Tancred $^{\mathrm{a}}$, Russel Perera ${ }^{\mathrm{a}}$, Jan Smital ${ }^{\mathrm{a}}$
}

\begin{abstract}
a Department of Physiology, Faculty of Medicine, Palacky University, Olomouc, Czech Republic,
${ }^{b}$ II $^{\text {nd }}$ Department of Medicine, University Hospital, Olomouc, Czech Republic,

c Department of Biophysics, Faculty of Medicine, Palacky University, Olomouc, Czech Republic. e-mail: rudolf.chlup@fnol.cz
\end{abstract}

Parts of this study were presented at the $28^{\text {th }}$ workshop of the AIDPIT Study Group, Igls, Austria, January 25 th $-27^{\text {th }}, 2009$

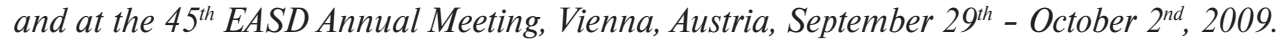

Received: August 13, 2009; Accepted: September 15, 2009

Key words: Glucose resorption/Buccal glucose spray/Dextrose tablets/Liquid sugars/Plasma glucose concentration

Objectives: The purpose of this prospective controlled trial was to assess the efficacy of three commercially available glucose products, (1) buccal glucose spray, (2) liquid sugars, and (3) dextrose tablet, on the evolution of plasma glucose concentration (PG).

Methods: Sixteen healthy volunteers aged $21.8 \pm 0.78$ y (mean \pm SE), BMI $23.5 \pm 0.84 \mathrm{~kg} / \mathrm{m}^{2}$, tested their PG over the course of 3 sets of 4 sessions $(\mathrm{S})$ each: $\mathrm{S}_{0}$-control fasting, $\mathrm{S}_{1}$-buccal administration of 10 glucose spray-doses $\left(0.84 \mathrm{~g}\right.$ of glucose) without swallowing; $\mathrm{S}_{2}$ consumption of 1 sachet $(13 \mathrm{ml})$ of liquid sugar (ca. $5.2 \mathrm{~g}$ glucose, $5.2 \mathrm{~g}$ fructose, $5.2 \mathrm{~g}$ sucrose); $\mathrm{S}_{3}$ consumption of one dextrose tablet $(6 \mathrm{~g})$. PG was tested in finger-prick capillary blood using a personal glucometer Linus at the start, and at 5, 10, 15, 20 and $30 \mathrm{~min}$. The means of 3 respective sessions for each of the 16 subjects were analyzed.

Results: The Wilcoxon signed rank test revealed no significant differences between changes in the mean PG at the start vs. 5-minute interval either in control, or any intervention sessions. Analysis of regression coefficients after $30 \mathrm{~min}$ compared to the control session, demonstrated an increase in PG with the sachet of liquid sugars $(0.068 \mathrm{mmol} / 1 / \mathrm{min}$, $\mathrm{p}=0.001)$ which was greater than a single dextrose tablet $(0.052 \mathrm{mmol} / 1 / \mathrm{min}, \mathrm{p}=0.002)$, but no significant $\mathrm{PG}$ increase was found after buccal glucose spray.

Conclusion: Liquid sugars or dextrose tablets, but not the buccal glucose spray, are effective means to increase PG within 10 minutes after ingestion.

\section{INTRODUCTION}

The oral mucosa is an attractive site for drug delivery in light of its high vascularity and its capacity for non-invasive drug administration. In addition, this drug delivery method bypasses the gastrointestinal tract, protects drugs and potentially other compounds such as glucose from degradation due to $\mathrm{pH}$, digestive enzymes in the gut, and/ or also from first-pass hepatic metabolism. Fast initiation and/or decline of delivery may result, and there is less inter-individual variability between patients ${ }^{1}$. Compared to transdermal delivery drug transport via mucosa is quicker due to its non-keratinized epithelium.

After Murrell sublingually used nitroglycerine in 1879 , there have been many attempts to use the oral mucosa not only for the delivery of other drugs, including insulin ${ }^{2}$, but also for the administration of glucose. In order to combat or avoid hypoglycaemia in persons with diabetes, the buccal glucose spray was believed to offer a sophisticated solution. In 2006, an easy-to-use buccal glucose rapid spray was developed. Reports were made on its immediate effects on hypoglycaemia and neuroglycopenia ${ }^{3,4}$, However, clear references to the effects of this buccal glucose rapid spray on plasma glucose concentration (PG) are unavailable. The purpose of this prospective controlled trial was to assess the efficacy of three commercially available glucose products (1) buccal glucose spray, (2) liquid sugars, and (3) dextrose tablet on the evolution of PG.

\section{SUBJECTS AND METHODS}

\section{Subjects}

Sixteen healthy volunteers (14 men and 2 women) aged $21.8 \pm 0.78$ y (mean \pm SE), BMI $23.5 \pm 0.84 \mathrm{~kg} / \mathrm{m}^{2}$, provided informed written consent and entered the study. The study was performed in accordance with the Helsinki Declaration of 1973 as revised in 2000 and approved by the local Ethics Committee.

\section{Study design}

The volunteers tested their PG over the course of three sequential sets of 4 sessions $\left(\mathrm{S}_{0}, \mathrm{~S}_{1}, \mathrm{~S}_{2}, \mathrm{~S}_{3}\right)$ each. In each set there was one control (fasting) session and 
three sessions testing different products of dextrose and other sugars:

- session $\mathrm{S}_{0}$ - control fasting;

- $\operatorname{session} \mathrm{S}_{1}$ - oral administration of 10 glucose spraydoses ( 5 doses on the mucosa of each cheek, as indicated by the manufacturer, without swallowing), i.e., $0.84 \mathrm{~g}$ of glucose at time $=0 \mathrm{~min}$;

- session $\mathrm{S}_{2}$ - consumption of one sachet of liquid sugars (13 ml, i.e., $20 \mathrm{~g}$ of jelly containing ca. $5.2 \mathrm{~g}$ glucose, $5.2 \mathrm{~g}$ fructose, $5.2 \mathrm{~g}$ sucrose and $4.4 \mathrm{~g}$ of water);

- session $\mathrm{S}_{3}$ - chewing and consumption of one dextrose tablet (6 g).

Each session was performed between 6 and 9 a.m., after an average fasting time of 8-9 hours (range was 6-13 hours). PG was tested in finger-prick capillary blood using a personal glucometer Linus, Wellion, Agamatrix, Inc., Salem, $\mathrm{NH}, \mathrm{USA}$, at the start $(\mathrm{t}=0 \mathrm{~min})$, at $\mathrm{t}=5$, 10, 15, 20 and 30 minutes. The glucose product, depending on the session, was used immediately after the first PG measurement. During all tests, the subjects remained seated, and did not engage in any physical activity.

\section{Statistical analysis}

Statistical software SPSS v. 15, SPSS Inc, Chicago, IL, USA, was used. In each of the 16 subjects, the means of 3 respective sessions (one session of each set) were calculated and analyzed. Shapiro-Wilk test of normality were performed and paired t-test or Wilcoxon signed rank test was used. Linear regression analysis ${ }^{5}$ was carried out to assess the evolution of PG in the course of the 30-minute fasting and after the consumption of tested dextrose products.

\section{RESULTS}

The 30-minute evolution of average PG in fasting state and after use of buccal glucose spray, dextrose tablets and liquid sugars is shown in Fig. 1.

The Wilcoxon signed rank test revealed no differences between the change of mean PG at the start vs. 5-minute intervals either in control- or in any intervention sessions (Fig. 2). Significant differences vs. start appeared as late as 10 min after the ingestion of liquid sugars or the dextrose tablet (Fig. 3) and increased after 15 and $20 \mathrm{~min}$. In addition, 20 minutes after consumption, the PG in the liquid sugars sessions were significantly higher than in the dextrose tablet sessions. Distribution of individual differences may be seen in the box graphs of Fig. 2 and Fig. 3.

After examining the whole 30-minute time period, analysis of regression coefficients for the change in $P G$ evolution in individual sessions demonstrated the highest increase after consumption of the liquid sugar $(0.068$ $\mathrm{mmol} / 1 / \mathrm{min}, \mathrm{p}=0.001)$. The single dextrose tablet had the second highest change in PG $(0.052 \mathrm{mmol} / 1 / \mathrm{min}$, $\mathrm{p}=0.002$ ). The change in PG levels for the buccal glucose spray was not significantly different from Controls (0.005 $\mathrm{mmol} / 1 / \mathrm{min}, \mathrm{p}=0.215)$. All interventions were

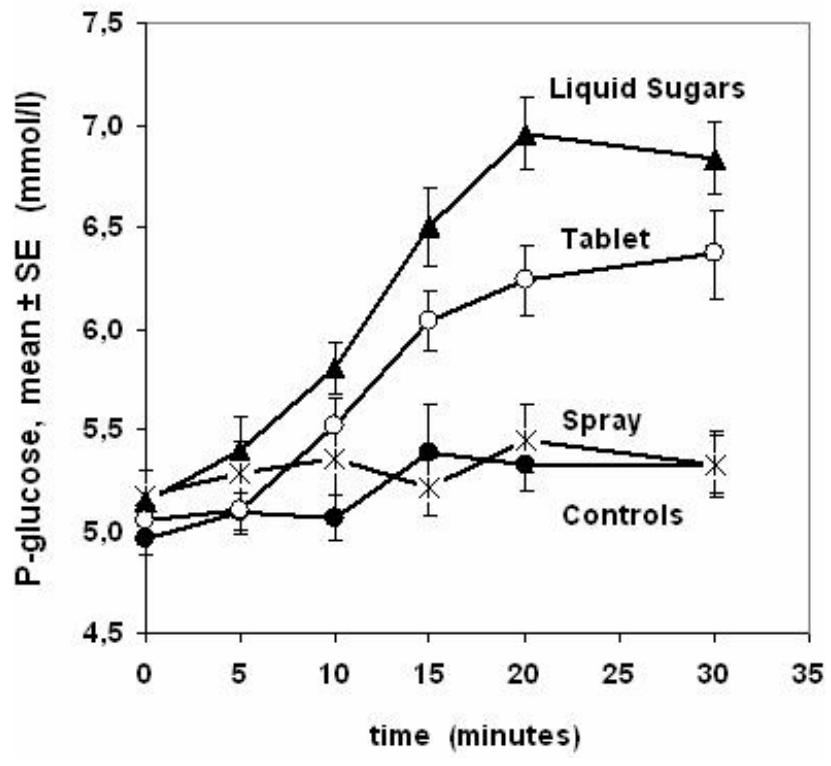

Fig. 1. Evolution of $P G$ in fasting state (Controls) and after use of glucose spray $(0.84 \mathrm{~g})$, one dextrose tablet $(6 \mathrm{~g})$ or liquid sugars (glucose $5.2 \mathrm{~g}$, fructose $5.2 \mathrm{~g}$ and saccharose $5.2 \mathrm{~g}$ ); $\mathrm{n}=16$.

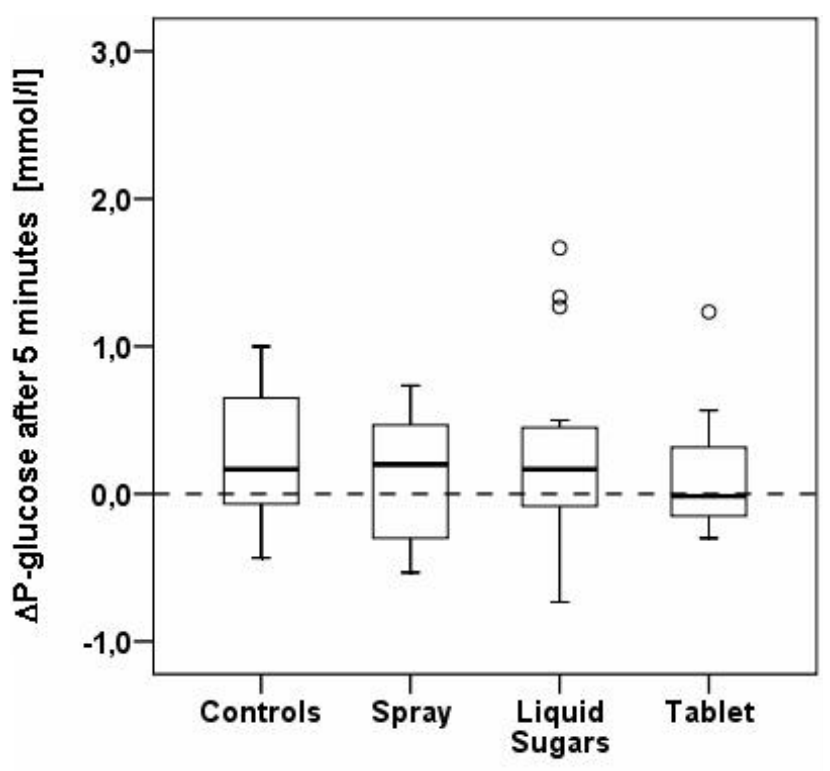

Fig. 2. Box graphs (median and quartiles): change of PG concentration after 5-min in fasting state (Controls) and after use of glucose spray (0.84 g), one dextrose tablet ( $6 \mathrm{~g}$ ) or liquid sugars (glucose $5.2 \mathrm{~g}$, fructose $5.2 \mathrm{~g}$ and saccharose $5.2 \mathrm{~g}$ ); $\mathrm{n}=16$; $\mathrm{o}=$ outliers. 


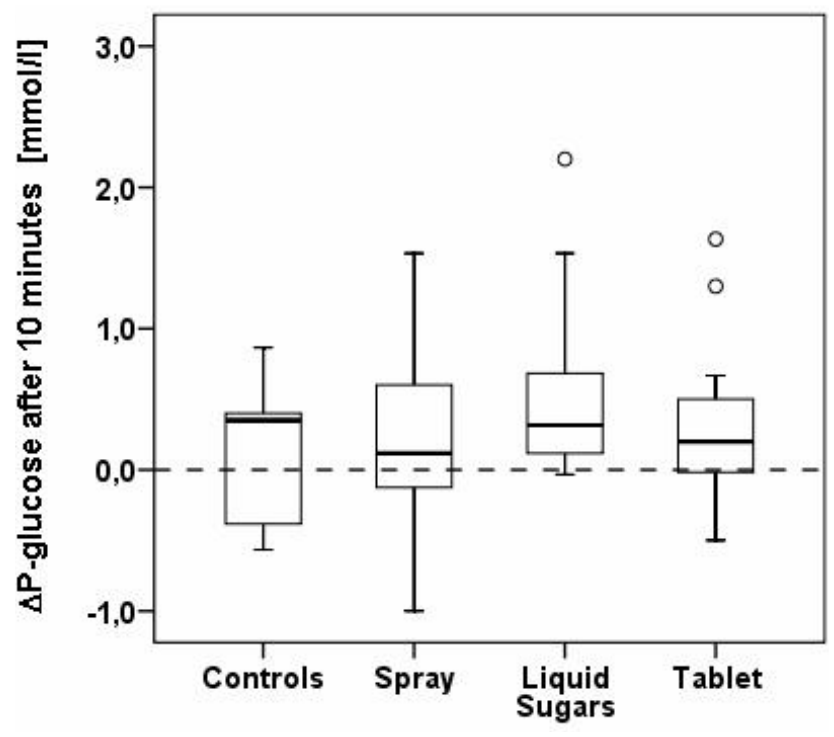

Fig. 3. Box graphs (median and quartiles): change of $P G$ concentration at 10-min interval in fasting state (Controls) and after use of glucose spray $(0.84 \mathrm{~g})$, one dextrose tablet $(6 \mathrm{~g})$ or liquid sugars (glucose $5.2 \mathrm{~g}$, fructose $5.2 \mathrm{~g}$ and saccharose $5.2 \mathrm{~g}) ; \mathrm{n}=16$; $\mathrm{o}=$ outliers.

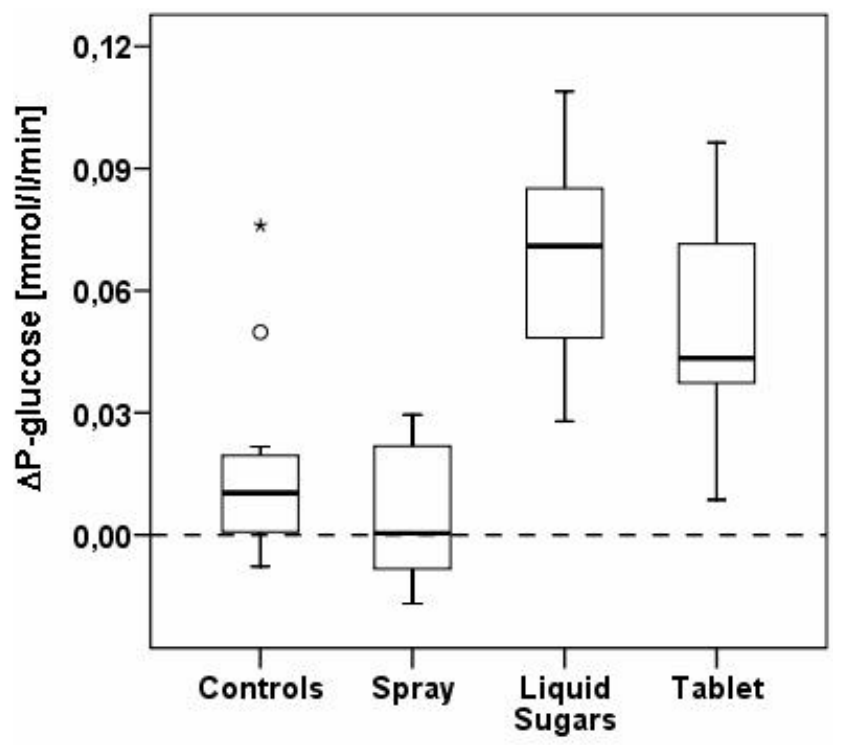

Fig. 4. Results of linear regression analysis. Box graphs (median and quartiles): rate of the increase of PG in individual sessions in the course of the whole 30-minute time period of fasting (Controls) and after use of glucose spray $(0.84 \mathrm{~g})$, one dextrose tablet $(6 \mathrm{~g})$ or liquid sugars (glucose $5.2 \mathrm{~g}$, fructose $5.2 \mathrm{~g}$ and saccharose $5.2 \mathrm{~g}$ ); $\mathrm{n}=16$; o = outliers; * = extreme values. compared to the control session (see box graphs in Fig. 4 for details).

\section{DISCUSSION}

Dextrose products are needed which are effective in increasing PG in hypoglycemia. Our study is focused on the potential benefits of glucose transport through the buccal mucosa and its comparison with other conventional forms of ingested glucose transport through the gut mucosa. As a control measure to avoid the influence of various endocrine and metabolic disturbances including hypo- and hyperglycaemia and effects of physical exercise, healthy fasting subjects were asked to remain in a sitting position and refrain from any excess movement.

In this study, no significant difference between the 30-minute PG evolution in the control session and in the session where buccal glucose spray, recommended by manufacturer and applied to the oral mucosa without swallowing, was demonstrated. These outcomes fully correspond to the results of others ${ }^{6,7}$. For example, Gunning and Garber's experiment with 2-Tritium labeled glucose in healthy non-diabetic volunteers which was published in the JAMA in 1978 in ref.7. They found the glycaemia increase via glucose absorption from the mouth to be less than $0.5 \mathrm{mg} / \mathrm{dl}(0.027 \mathrm{mmol} / 1)$, and no change in serum insulin level. They concluded that significant buccal absorption of glucose does not occur in man, and that instant glucose appears to be of therapeutic value only if swallowed by fully conscious hypoglycaemic patients, that is, it would not benefit unconscious patients because of its poor absorption through the buccal mucosa ${ }^{7}$.

On the other hand, in our study both the liquid sugars and the dextrose tablet resulted in a significant increase in PG within 10 minutes of swallowing.

Our results are undoubtedly weakened by the fact that the amounts of dextrose, fructose and saccharose in the products were different. Nevertheless, from the point of view of final hyperglycaemic effect of a dextrose product being used according to manufacturer's recommendation, this argument does not seem to be of practical importance.

The question arises what glucose concentration may be reached when dissolving the tested amounts of glucose in circulating blood. The applied dose of buccal glucose spray contained $0.84 \mathrm{~g}$ glucose. If we dissolve the $0.84 \mathrm{~g}$ (i.e. $4.7 \mathrm{mmol}$ ) of glucose in 5.01 of blood the maximum increase in glucose concentration would be $4.7 / 5.0=$ $0.94 \mathrm{mmol} / 1$. Taking into consideration continuous glucose uptake and flux in various tissues, the final increase in PG is expected to be somewhat below this rate. In our experiments the mean and maximum PG increase 5 minutes after the use of buccal spray was $0.098 \mathrm{mmol} / 1$ and $0.733 \mathrm{mmol} / 1$, respectively, whereas in controls these values were not significantly different $(0.127 \mathrm{mmol} / \mathrm{l}$ and $0.700 \mathrm{mmol} / 1$, respectively) - see Fig. 1 and Fig. 2 .

One tested dextrose tablet comprised $6 \mathrm{~g}$ of glucose. If we dissolve $6 \mathrm{~g}$ (i.e. $33.6 \mathrm{mmol}$ ) in 5.01 of solute (blood) 
the maximum glucose concentration would be 33.6/5.0= $6.7 \mathrm{mmol} / 1$. Taking into consideration the glucose uptake and flux in various tissues as in the previous example, the real attainable PG increment would be less.

Apart from the variable quantity of sugars in tested products, the discrepancy between the maximum expected and realized buccal vs. intestinal increase in glucose concentration could be due to the different absorption rates of buccal and intestinal mucosa.

Oral mucosa. According to the up-to-date concept of glucose and other drugs absorption via oral mucosa, there are two possible routes:

1. Transcellular route (passing through the cell): The transport of food sugars across human buccal mucosa shows proven stereospecifity. For the natural isomers D-glucose and D-galactose the transport is at least partly sodium-dependent ${ }^{8,9}$. The presence of a specialized sodium ion dependent transport mechanism for D-glucose was found in the dorsum of the tongue and (with slower absorption) from the ventral surface of the tongue ${ }^{10,11}$. Further, as a result of the in vitro uptake study using specific inhibitors, it has been suggested that SGLT1, GLUT1, GLUT2 and GLUT3 are expressed in the epithelial cells of human oral mucosa and take part in the glucose uptake ${ }^{12}$. Sugar transport functions not only to take up D-glucose but also to transport it across the stratified epithelial layer ${ }^{13}$.

2. Paracellular route (passing around the cell): This second way is most interesting from the point of view of our topic ${ }^{1}$. Absorption and transport are affected by charge, conformation and lipophilicity ${ }^{2}$.

Smaller molecules exhibit rapid transport across the mucosa. The more lipid-soluble the molecule is, the higher the permeability. Hydrophilic macromolecules such as peptides require absorption enhancers to overcome their low permeability.

It was assumed that the process of penetration of different compounds across oral mucosa is most likely by simple diffusion. Although an active transport of sodium has been found the amount was too small to be considered a quantitatively important process ${ }^{14,15}$.

Studies with microscopically visible tracers ${ }^{14}$ indicated that a major route across stratified epithelium for many compounds is the intercellular space. However, a barrier for penetration was identified in the intercellular substance in the superficial layers of epithelium. This barrier was theorized to be of a lipid nature. The presence of membrane-coating granules in stratified squamous epithelium was found to be strategic for the formation of this permeability barrier which was proved to not be absolute, even for relatively large molecules.

The rates at which different compounds can penetrate epithelium depend on their size and chemical nature. It is also assumed that the salivary mucin may contribute to the permeability barrier ${ }^{14}$. For this reason, neither the transcellular nor paracellular route have adequate capacity to increase the rate of glucose transport across oral mucosa.
Intestinal mucosa. The essential physiological absorption tissue for glucose is the gut. Analogically, in the gut there are two possible routes for glucose absorption.

1. Transcellular absorption via the $\mathrm{Na}+$ /glucose cotransporter SGLT1 which transports D-glucose against its concentration gradient into the enterocyte, where it is used for its own metabolic needs, or transported by the facilitative sugar transporter GLUT2 across the basolateral membrane ${ }^{16}$.

2. Paracellular glucose absorption. Pappenheimer et al. proposed the paracellular solvent drag between absorptive cells contributes a passive component to absorption, which, at high sugar concentrations, such as in the jejunal lumen after a meal and after saturation of membrane carriers, is much more utilized than the active transport through SGLT1 (60-80\% of total absorption $)^{17,18}$.This paracellular transport increases exponentially with body weight and may account for the capacity of human intestines to absorb large amounts of glucose during glucose tolerance tests, prolonged exercises, and oral glucose-saline rehydratation therapy ${ }^{17}$. Kellet et al believe that this phenomenon considered as a non-carrier mediated transport is in fact a facilitated transport mediated by transient insertion of glucose transporter type 2 GLUT2 in apical membrane when glucose concentration in lumen exceeds the SGLT1 transport capacity ${ }^{19-22}$.

Our study demostrated that 0.84 grams of glucose administered via buccal glucose spray into the buccal membrane was ineffective at increasing the PG in healthy volunteers without hypoglycaemia. Nevertheless, the experience of other groups $s^{3,4,23-25}$ that glucose spray applied to buccal membrane provides immediate relief of hypoglycaemic symptoms and that frequent use of a buccal glucose spray over 6 months in small children resulted in improved HbA1c, cannot be neglected. To explain these contradictory findings, several factors should be taken into consideration.

First, as demonstrated by Manish Wani ${ }^{1}$ buccal absorption of drugs may be very fast initially but also result in a quick decrease in blood concentrations of these drugs. Concurrently, we measured blood glucose levels at 5-minute intervals and any changes in glycaemia occurring in the interim would have been missed. Considering the delay in venous blood reaching finger capillaries (up to approximately 1 minute), the collected glycaemia measurements are not perfectly synchronized with buccal mucosa glucose absorption. Thus it is theoretically possible that the PG levels did rise and then fell very quickly.

On the other hand, regarding both the transcellular and paracellular routs of glucose transport across the oral mucosa (see above), this hypothesis does not seem to be valid for glucose. Independently, in the course of chewing a dextrose tablet or having liquid sugars in mouth before swallowing, the dextrose has excellent access to buccal membranes leaving the question what should be the advantage of glucose spray?

Finally it is possible that glucose is simply not effectively taken up by the oral mucosa membrane and a po- 
tential placebo- and/or study-in effects must be taken in consideration.

\section{CONCLUSIONS}

This study demonstrates the efficacy of dextrose tablet and liquid sugars as means of increasing glycaemia within 10 minutes of ingestion. The buccal glucose spray does not appear to be efficacious with its current composition. In light of these findings, we prefer the use of tablets or liquid sugars for the purpose of combating hypoglycemia, as opposed to the buccal glucose spray. Further studies on the pharmacokinetics of buccal glucose are necessary.

\section{ACKNOWLEDGEMENTS}

Supported by the grants IGA NR 7825-3, IGA 10823, Ministry of Health, and MSM 6198959216, Ministry of Education, Czech Republic.

\section{REFERENCES}

1. Manish Wani S. Current Status In Buccal Drug Delivery System Pharmaceutical Reviews (e-journal), Latest Reviews 2007; 5(2) Available from: http://www.pharmainfo.net/reviews/current-statusbuccal-drug-delivery-system.

2. Bernstein G. Delivery of insulin to the buccal mucosa utilizing the Rapid Mist system. Expert Opin Drug Delivery 2008; 5(9): 10047 10055.

3. Picardi A, Merola YM, Altomare M, Visalli N, Cipponeri E, Pozzilli P. Glucose Rapidspray - a new tool to control progression towards hypoglycemia. Diabetes 2007; 56(1): A128 (Abstract).

4. Khazrai M, Cacciapaglia M, Picardi A, Anguissola GB, Guglielmi $\mathrm{C}$, Manfrini S et al.Treatment of symptoms of neuroglycopenia with glucose Rapid Spray to avoid rebound hyperglycemia. Diabetes 2008; 57(1):A564 (Abstract).

5. Langova K, Pribylova H, Kajabova M, Luza J. Assessment of haemoglobin A1c evolution using various statistical methods (survival analysis and linear regression) in persons with diabetes mellitus. Biomed Pap Med Fac Univ Palacky Olomouc Czech Rep. 2009;153 (2):137-144.

6. Breuninger H, Feine U. On the uptake of labeled glucose by the mucous membranes of the nose, mouth and middle ear. Arch Klin Exp Ohren Nasen Kehlkopfheilkd 1968; 191(2): 662-665.

7. Gunning RR, Garber AJ. Bioactivity of Instant Glucose - Failure of Absorbtion Through Oral Mucosa. JAMA 1978; 240(15): 16111612 .
8. Manning AS, Evered DF. The absorbtion of sugars from the human buccal cavity, Clin Sci Mol Med 1976;52,127 - 132.

9. Sadoogh - Abasian F, Evered DF . Absorption of vitamin C from the human buccal cavity. Br JNutr 1979; 42: 15-20.

10. Kurosaki Y, Yano K, Kimura T. Perfusion Cells for Studying Regional Variation in Oral Mucosal Pemeability in Humans. J Pharm Sci 1998; 87(5): 613- 615.

11. Gherzi R, Melioli G, De Luca M, D‘Agostino A, Guastella M, Traverso CE et al. High expression levels of the erythroid/brain type glucose transporter (GLUT1) in the basal cells of human eye conjunctiva and oral mucosa reconstituted in culture. Exp Cell Res 1991; 195 (1): 230-236.

12. Oyama Y, Yamano H, Ohkuma A, Ogawara K, Higaki K, Kimura T. Carrier- mediated transport systems for glucose in mucosal cells of the human oral cavity. J Pharm Sci 1999; 88(8): 830-834.

13. Kimura T, Yamano H, Tanaka A, Matsamura T, Ueda M, Ogawara K., Higaki K. Transport of D-glucose across cultured stratified cell layer of human mucosal cells. J Pharm Pharmacol 2002; 54(2): 213-219.

14. Squier CA. The permeability of oral mucosa. Crit Rev Oral Biol Med 1991; 2(1):13-32.

15. Reid CO, Hardcastle J, Smith CJ. A comparison of the permeability characteristics of intact and tape-stripped hamster cheek pouches in vitro. J Dent Res 1986; 65(5): 673 - 676

16. Koeppen BM, Stanton BA. Berne,Lévy PHYSIOLOGY, 6th edition MOSBY Inc. 2008

17. Pappenheimer JR. On the coupling of digestion with intestinal absorption of sugars and amino acids. Am J Physiol 1993; 265 (3): 409-416.

18. Pappenheimer JR. Paracellular intestinal absorption of glucose,creatinine and mannitol in normal animals: relation to body size, Am J Physiol 1990; 259(2): 290-299.

19. Helliwell PA, Kellett GL. The active and passive components of glucose absorption in rat jejunum under low and high perfusion stress. J Physiol (Lond) 2002; 544(2): 579-589.

20. Kellet GL, Brot-Laroche E. Apical GLUT2- A Major Pathway of Intestinal Sugar Absorption. Diabetes 2005; 54: 3056-3062.

21. Kellett GL. The facilitated component of intestinal glucose absorption. J Physiol (Lond), 2001; 531(3): 585-595.

22. Kellett GL. Stress and intestinal sugar absorption, J Physiol Regul Integr Comp Physiol 2007; 292 (2): 860 - 861.

23. Khazrai YM, Altomare M, Visalli N, Cipolloni L, Maurizi AM, Pozzilli P. Reduction of body weight through diet and the use of Glucose Rapidspray. Diabetes 2007; 56(1):A463 (Abstract).

24. Pronina E, Petraikina H, Antsiferov M, Duchareva O, Gugliemi C, Khazrai M, Pozzilli P.Glucose Rapidspray for a new management of hypoglycaemia in children up to 5 years of age with type 1 diabetes mellitus. Diabetologia 2008; 51(Suppl 1): S383 (Abstract).

25. Pronina E, Gugliemi C, Petraikina H, Antsiferov M, Duchareva O, Pozzilli P. A novel tool to kontrol hypoglycaemia and improve HbA1c in very young children with type 1 diabetes mellitus. Diabetes 2009; 58(Suppl 1): A457(Abstract). 
